\title{
TEMPO DE INTERNAÇÃO E A OCORRÊNCIA DE EVENTOS ADVERSOS A MEDICAMENTOS: UMA QUESTÃO DA ENFERMAGEM
}

\author{
The length of stay and the occurrence of adverse drug: a question of nursing \\ Tiempo de internación y la ocurrencia de eventos adversos por medicamentos: una \\ cuestión de la enfermería
}

Enirtes Caetano Prates Melo²

\begin{abstract}
RESUMO
0 objetivo do estudo foi estimar o efeito do tempo e das características individuais na ocorrência de evento adverso a medicamentos em pacientes com afecções cardiológicas. Trata-se de um estudo avaliativo sobre a ocorrência de evento adverso a medicamentos realizado em um hospital público e cardiológico, localizado no Município do Rio de Janeiro. Para análise da sobrevida utilizou-se o método de Kaplan-Meier. A probabilidade de sobreviver livre de evento adverso a medicamentos até 30,60 e 100 dias foi respectivamente de $96 \%, 93 \%$ e $73 \%$. Os eventos adversos a medicamentos representam condições marcadoras que descrevem o desempenho dos serviços de saúde. A detecção de eventos adversos nas instituições hospitalares possibilita conhecer as falhas que ocorrem no sistema de medicação e ainda implementar estratégias para reduzi-las.
\end{abstract}

Palavras-chave: Monitoramento de Medicamentos. Qualidade da Assistência à Saúde. Análise de Sobrevida. Enfermagem.

\begin{abstract}
The objective of the study was to estimate the time effect and the individual characteristics in the occurrence of an adverse event to drugs in patients with cardiac diseases. It is an evaluative study about the occurrence of an adverse event to drugs that took place at a public and cardiologic hospital, located in the city of Rio de Janeiro. For the analysis of the survival, the method Kaplan-Meier was used. The probability of surviving free from an adverse event to drugs up to 30,60 and 100 days was respectively of $96 \%, 93 \%$ and $73 \%$. The adverse events to drugs represent marking conditions that describe the performance of the health services. The detection of adverse events in hospitals makes it possible to know the failures that occur in the medication system and also to implement strategies to reduce them.
\end{abstract}

Keywords: Drug monitoring. Quality of health care. Survival analysis. Nursing.

\section{Resumen}

El objetivo del estudio fue estimar el efecto del tiempo y de las diferencias individuales en la ocurrencia de eventos adversos por medicamentos en pacientes con enfermedades cardíacas. Este es un estudio de evaluación sobre la presencia de evento adverso a cabo en un hospital público y cardiología, ubicado en Río de Janeiro. Para el análisis de supervivencia se utilizó el método de Kaplan-Meier. La probabilidad de supervivencia libre de eventos adversos por medicamentos por 30, 60 y 100 días fueron respectivamente del $96 \%, 93 \%$ y $73 \%$. Los eventos adversos por medicamentos representan condiciones marcadoras que describen el desempeño de los servicios de salud. La detección de eventos adversos en los hospitales hace que sea posible conocer los errores que ocurren en el sistema de medicación, así como aplicar estrategias para reducirlos.

Palabras-clave: Monitoreo de drogas. Calidad de la atención de la salud. Análisis de supervivencia. Enfermería. 


\section{INTRODUÇÃO}

A qualidade na assistência e a segurança do paciente são metas a serem atingidas pelos profissionais e instituições de saúde. No entanto, apesar de esforços no sentido de alcançar um cuidado de qualidade, livre de riscos e falhas, convive-se com inúmeras ocorrências de eventos adversos a medicamentos (EAMs). Tal fato, compromete a efetividade do cuidado e pode ocasionar dano ao paciente. A ocorrência do dano em consequência de um EAM pode agravar seu quadro clínico, aumentar os custos para as instituições e sociedade ou conduzir ao óbito. ${ }^{1-2}$

Os custos diretos relacionados à ocorrência de EAM ocasionam altos gastos decorrentes da execução de exames adicionais, aumento significativo da permanência hospitalar, uso de medicamentos suplementares, necessidade de realização de procedimentos médicos e, ainda, a transferência para unidades de terapia semi-intensiva/intensiva. Além destes, devemos considerar os custos indiretos associados à perda da produtividade e ao sofrimento físico e psicológico do paciente e familiares..$^{3-4}$

Alguns estudos descrevem que idade avançada, sexo, comorbidades, polifarmácia, uso inapropriado de medicamentos e ilegibilidade nas prescrições são os principais fatores de risco associados aos EAMs. ${ }^{1,5-7}$ Fatores como raça/etnia, renda e nível de escolaridade podem influenciar na evitabilidade do evento, no entanto são pouco estudados. ${ }^{1}$

Insuficiência cardíaca congestiva, doença vascular periférica, doença pulmonar crônica, doença reumatológica, insuficiência hepática, insuficiência renal, diabetes mellitus e câncer possuem estreita relação com 0 aumento das readmissões hospitalares de pacientes por reações adversas a medicamentos. $^{6}$

Um estudo de caso-controle realizado em Uthah analisou 91.574 pacientes e constatou que 2.227 apresentaram EAM durante a internação hospitalar; isso corresponde a uma taxa de 2,43 EAM por 100 admissões. Identificou adicionalmente um aumento no tempo de internação hospitalar entre os casos com EAM e os controles (7,69 vs 4,46 dias). ${ }^{8}$

É fato que o tempo de permanência de pacientes com afecções cardiológicas tem diminuído substancialmente durante as últimas três décadas. Essa redução está relacionada a uma melhor compreensão do processo de adoecimento, aos avanços nos procedimentos diagnósticos e terapêuticos, ao aprimoramento de estratégias de reabilitação do paciente externo (de ambulatório) e, recentemente, às pressões econômicas. Pesquisas têm mostrado um baixo risco de resultados adversos em pacientes sem maiores complicações clínicas, no período de 3 a 5 dias após o infarto agudo do miocárdio, por exemplo. ${ }^{9} 0$ prolongamento da internação, em pacientes com um curso não complicado da doença, pode representar um fator adicional para a ocorrência de EAM. Este estudo teve como objetivo estimar o efeito do tempo e das características individuais na ocorrência de EAM em pacientes com afecções cardiológicas.

\section{METODOLOGIA}

Trata-se de um estudo avaliativo sobre a ocorrência de EAM realizado em um hospital público e especializado em cardiologia (denominado Hospital Y), localizado no Município do Rio de Janeiro. A pesquisa constou de duas fases distintas, sendo a primeira a identificação e análise dos EAMs através de adaptação do método proposto pelo Institute for Healthcare Improvement $(\mathrm{IHI}),{ }^{10}$ baseado em uma revisão retrospectiva de prontuários orientada por critérios explícitos de rastreamento. Na segunda foi estimado o efeito do tempo na ocorrência de EAM por meio da técnica de análise de sobrevida.

Para fins desse estudo, EAM foi considerado como qualquer ocorrência médica desfavorável, que pode ocorrer durante 0 tratamento com um medicamento, mas que não possui, necessariamente, relação causal com esse tratamento. ${ }^{11}$

A base de dados foi composta por pacientes internados no hospital relacionado, no período de dezembro de 2007 a fevereiro de 2008, incluindo maiores de 18 anos que permaneceram internados por pelo menos 48 horas. Foram excluídos pacientes provenientes da obstetrícia e aqueles que estivessem em tratamento oncológico.

Foram elegíveis para o estudo 551 prontuários, sendo extraída uma amostra aleatória simples de 112 prontuários, estimada a partir dos seguintes critérios: erro de 10\%, significância estatística de 95\%, prevalência do evento estudado de $10 \%$ e perdas de $10 \%$. 0 tamanho amostral está associado ao nível de precisão desejado. Nesse estudo o erro de amostragem considerado tolerável para estimar a prevalência de EAM foi compatível com aquele arbitrado em outros estudos da área. ${ }^{12} \mathrm{~A}$ seleção aleatória dos prontuários foi realizada a partir da listagem mensal de alta, obtida junto ao Serviço de Admissão e Alta do hospital.

Apesar de os pacientes terem sido acompanhados até a alta hospitalar, consideraram-se para efeito de análise apenas os primeiros 50 dias após a internação. Essa medida teve 0 objetivo de evitar a possibilidade de viés nas estimativas de sobrevida. Recomenda-se, nos estudos de sobrevida em que 0 período de observação é extenso, assegurar que não tenha havido mudanças importantes nas características dos indivíduos sob estudo e no diagnóstico ao longo do período. ${ }^{13}$

0 tempo de sobrevida foi calculado considerando 0 intervalo de tempo desde a entrada do paciente no estudo (data de admissão no hospital) até a ocorrência do EAM ou de censura (observação superior 50 dias).

Os diagnósticos de admissão foram organizados em três grupos para o estudo da sobrevida: doenças isquêmicas do coração, outras doenças cardíacas e outras doenças. 0 grupo de doenças isquêmicas do coração incluiu as seguintes patologias: angina, infarto agudo do miocárdio e doença 
coração. Endocardite aguda e subaguda, cardiomiopatias, insuficiência cardíaca, arritmias cardíacas, transtornos não reumáticos da valva mitral e aórtica, cardiomiopatias, endocardite de valva etranstorno da valva pulmonarforam as patologias incluídas no grupo de outras doenças cardíacas. 0 grupo "outras doenças" foi composto por: anormalidades do batimento cardíaco, aneurisma e dissecção da aorta, malformações congênitas das grandes artérias, malformaç̧ões congênitas das valvas aórtica e mitral, doença reumática da valva mitral e aórtica, edema agudo do pulmão, doença de chagas, embolia e trombose arteriais, traumatismo craniano, febre de origem desconhecida e reação anormal em paciente ou complicação tardia causada por procedimentos médicos.

As variáveis estudadas foram: sexo, idade, escolaridade, ocupação, raça/etnia, caráter da admissão, diagnóstico de admissão, comorbidades, diagnóstico de saída e local de ocorrência do EAM. Utilizou-se o método de Kaplan-Meier na análise exploratória das variáveis disponíveis.

0 banco de dados foi gerado em programa computacional MS-Access, desenvolvido para essa pesquisa. Para a análise estatística, a base de dados foi exportada e processada com o auxílio do programa SPSS ${ }^{\circledR}$ versão 13.0.

0 projeto de pesquisa $n^{0}$ 0192/22.02.2008 foi submetido e aprovado pelo Comitê de Ética e Pesquisa do hospital estudado para a averiguação dos aspectos éticos da pesquisa e consequente autorização para a realização do estudo, segundo a Resolução 196/96, que regulamenta pesquisas em seres humanos. Não há conflito de interesses no estudo.

\section{RESULTADOS}

Foram avaliados 112 pacientes elegíveis dentre aqueles internados no período sob estudo. Houve a confirmação de $16 \operatorname{EAM}(14,3 \%)$, ocorridos em 15 casos.

Dos casos que apresentaram eventos adversos, verificou-se uma pequena predominância do sexo masculino (66,7\%); a média de idade foi 59 anos (desvio-padrão \pm 15,82) (Tabela 1). A elevada proporção de informação ignorada no caso das variáveis referentes à raça/etnia $(27,7 \%)$ e escolaridade (67\%) comprometeu a análise dessa dimensão, com problemas no registro dos profissionais de saúde no prontuário do paciente.

Metade das internações analisadas (50\%) foi eletiva, apresentando um tempo médio de internação considerado elevado (média de 18,27 dias, desvio-padrão 20,18), variando de 2 a 138 dias (Tabela 1). Esse tempo pode variar de acordo com as características dos pacientes e do hospital, o tipo de remuneração da internação e a disponibilidade de tratamentos alternativos (como o caso do tratamento domiciliar). No caso do infarto agudo do miocárdio, por exemplo, considera-se aceitável um tempo mediano de permanência entre 7 a 11 dias. ${ }^{14}$

Tabela 1: Características demográficas dos pacientes e da internação no Hospital Y, Rio de Janeiro, dezembro de 2007 a fevereiro de 2008

\begin{tabular}{|c|c|}
\hline Cara cterísticas demográficas e da inte rn ação & Frequência \% (n) \\
\hline \multicolumn{2}{|l|}{ Sexo } \\
\hline Masculin o & $50,9 \%(57)$ \\
\hline Feminino & $49,1 \%(55)$ \\
\hline \multicolumn{2}{|l|}{ Faixa Etária } \\
\hline Até 59 anos & $50,9 \%(57)$ \\
\hline 60 anos ou mais & $49,1 \%(55)$ \\
\hline Idade Média: 58,4 anos (desvio-padrão \pm & \\
\hline \multirow{2}{*}{\multicolumn{2}{|c|}{$\begin{array}{l}\text { Mediana: } 59 \text { anos } \\
\text { Caráter da Admissão }\end{array}$}} \\
\hline & \\
\hline Eletiva & $50 \%(56)$ \\
\hline Urqência & $39.3 \%(44)$ \\
\hline Emergência & $10,7 \%(12)$ \\
\hline \multicolumn{2}{|l|}{ Tempo de Internação } \\
\hline $2-4$ dias & $25,9 \%(29)$ \\
\hline $5-12$ dias & $25 \%(28)$ \\
\hline $13-25$ dias & $25 \%(28)$ \\
\hline Mais de 25 dias & $24,1 \%(27)$ \\
\hline \multirow{2}{*}{\multicolumn{2}{|c|}{$\begin{array}{l}\text { Tempo Médio: } 18,27 \text { dias (desvio-padrão } \pm \\
\text { Mediana: } 12 \text { dias }\end{array}$}} \\
\hline & \\
\hline \multicolumn{2}{|l|}{ Diagnóstico de Admissão } \\
\hline Anqina & $27,7 \%(31)$ \\
\hline Insuficiên cia Cardíaca & $17,9 \%(20)$ \\
\hline Arritmias & $11,6 \%(13)$ \\
\hline Infarto Aqudo do Mio cárdio & $10,7 \%(12)$ \\
\hline Cardio m iopatia & $8,9 \%(10)$ \\
\hline Estenose de Mitral e Aorta & $8 \%(9)$ \\
\hline Doença Isquêmica & $5,4 \%(6)$ \\
\hline Outros & $9,8 \%(11)$ \\
\hline \multicolumn{2}{|l|}{ Comorbidades } \\
\hline 1-3 patologias & $42 \%(47)$ \\
\hline Mais de 4 patologias & $55,4 \%(62)$ \\
\hline Nenhum a & $2,7 \%(3)$ \\
\hline \multicolumn{2}{|l|}{ Condição de Saída } \\
\hline Morto & $6,3 \%(7)$ \\
\hline Vivo & $93,8 \%(105)$ \\
\hline
\end{tabular}


Os principais diagnósticos de admissão foram angina (27,7\%), seguida de insuficiência cardíaca (17,9\%) e arritmias cardíacas (11,6\%). Dos casos, 55,4\% apresentavam mais de quatro patologias associadas. Dos pacientes, $34,8 \%$ eram tabagistas, $10,7 \%$ obesos e $4,5 \%$ etilistas.

0 tempo médio de internação foi mais baixo entre as mulheres, os mais jovens ( 21 a 39 anos) e aqueles que apresentavam um menor número de comorbidades (abaixo de quatro patologias).

Entre os casos estudados, o número de EAM apresentou relação com o tempo de internação. Entre 2 a 4 dias de internação foram identificados quatro eventos adversos. Em períodos mais longos de internação (mais de 26 dias) foram confirmados cinco eventos adversos.

A probabilidade de sobreviver livre de EAM até 30 , 60 e 100 dias foi respectivamente de $96 \%, 93 \%$ e $73 \%$. As curvas de sobrevida (estimativas Kaplan-Meier) apresentaram comportamento diferenciado desde o início do seguimento para a variável sexo. Nos períodos mais curtos de internação os pacientes do sexo masculino têm uma sobrevida mais elevada. No entanto, a partir de 30 dias este padrão se inverte (Figura 1).

Figura 1: Kaplan-Meier das internações com EAM, Hospital Y, Rio de Janeiro, dezembro de 2007 a fevereiro de 2008

Sobrevida segundo o sexo

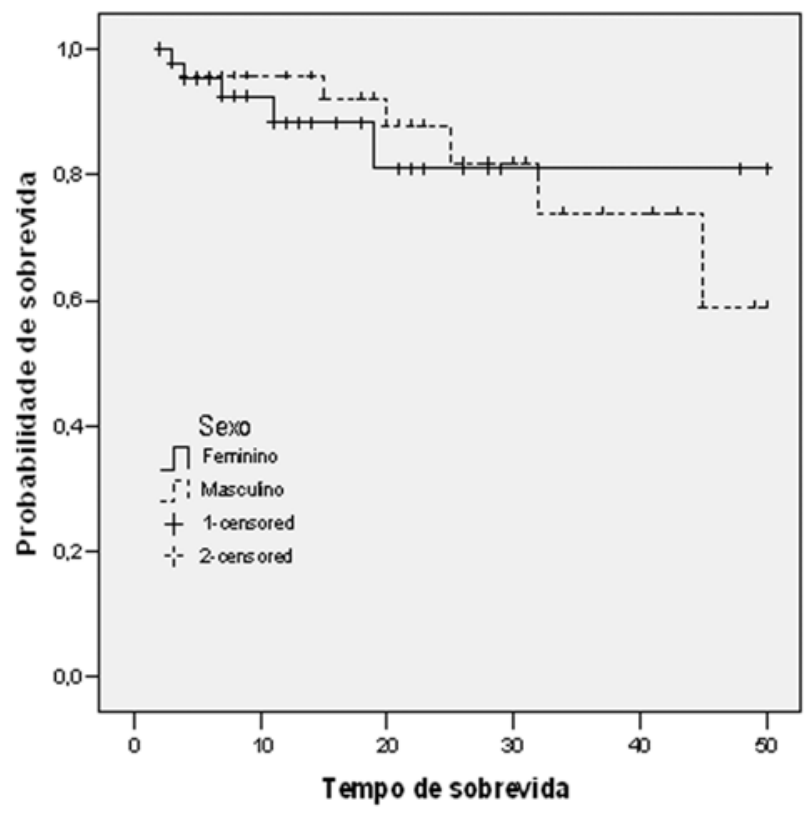

Sobrevida segundo a faixa etária

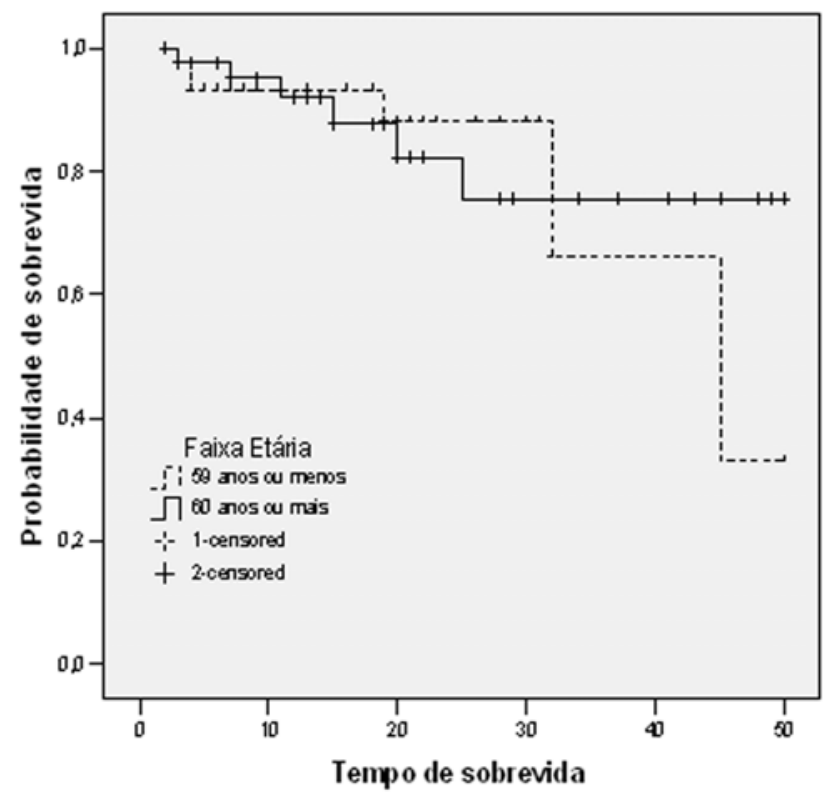


Não se observou variação na sobrevida livre de EAM em relação aos grupos etários mais jovens, diferença notada somente entre aqueles acima de 60 anos (Figura 1). Não se observou variação relevante na sobrevida livre de EAM em relação ao diagnóstico de admissão do paciente. Ainda assim, pacientes admitidos em decorrência de doenças isquêmicas cardíacas parecem apresentar uma sobrevida um pouca mais elevada. No entanto, é nítido o gradiente entre os grupos segundo o diagnóstico de alta, em que a sobrevida de pacientes com doenças isquêmicas do coração é claramente mais elevada, mesmo entre os tempos mais longos (Figura 2).

Cabe destaque a distribuição da sobrevida em relação ao caráter da admissão. Há uma variação da probabilidade de ocorrência de evento adverso ao longo do tempo, provavelmente relacionada à gravidade dos casos. Pacientes admitidos em caráter de urgência/emergência apresentaram inicialmente sobrevida livre de EAM mais elevada, padrão que se inverte após 20 dias de internação (Figura 2).

Figura 2: Kaplan-Meier das internações com EAM, Hospital Y, Rio de Janeiro, dezembro de 2007 a fevereiro de 2008

Sobrevida segundo o caráter de admissão
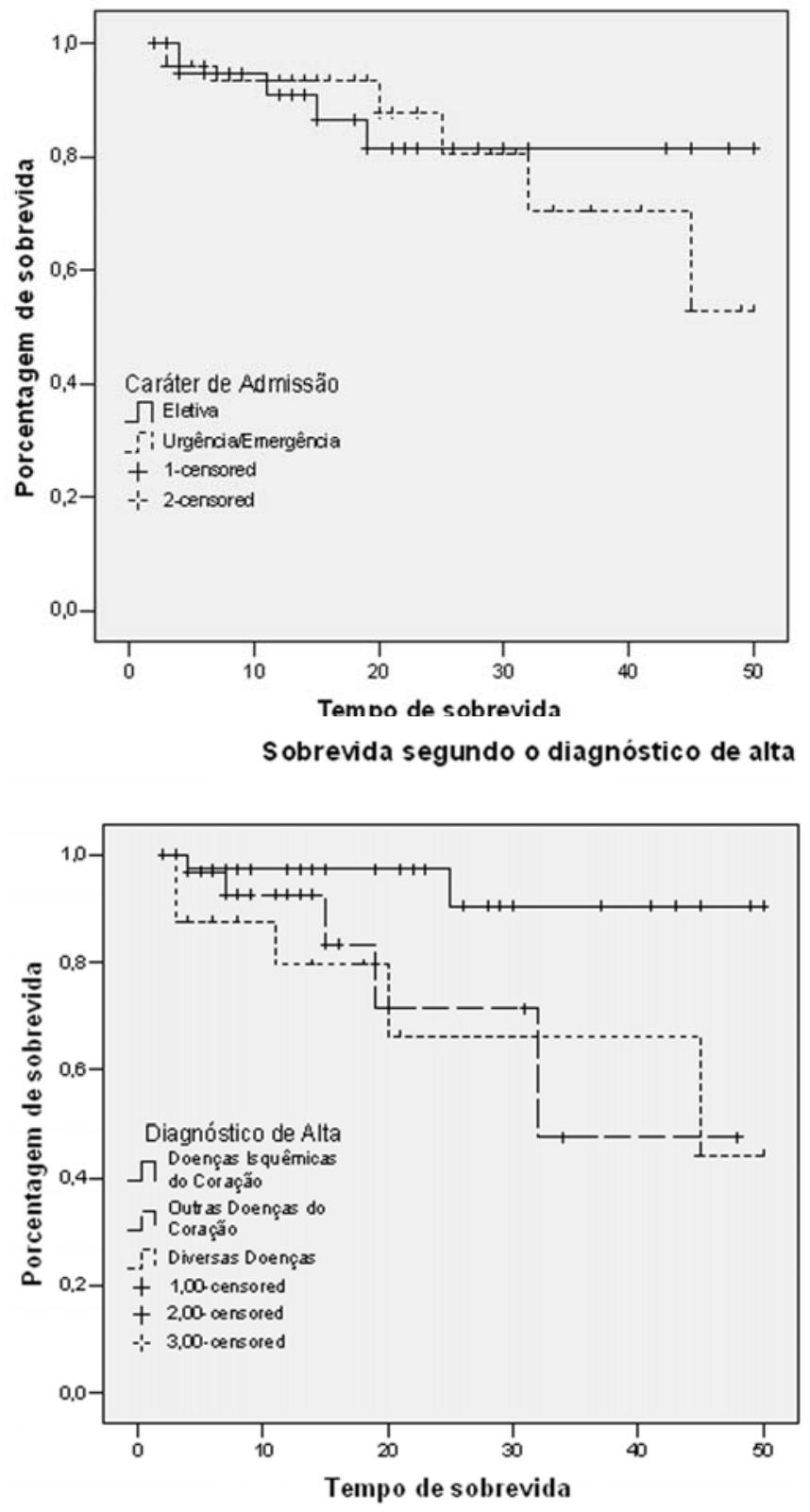
Os EAMs concentraram-se em unidade de terapia intensiva $(6,3 \%)$, enfermaria $(2,7 \%)$, centro cirúrgico $(1,8 \%)$, residência do paciente $(1,8 \%)$ e unidade de pronto atendimento $(0,9 \%)$. Não houve variação significativa na sobrevida livre de EAM em relação ao local de ocorrência do evento.

\section{DISCUSSÃO}

Nesse estudo observou-se uma alta incidência de EAM (14,3\%). 0 evento adverso deve ser entendido como um evento sentinela, sendo fundamental a compreensão de como e o porquê de sua ocorrência.

No Rio de Janeiro, dois importantes estudos identificaram a ocorrência de EAMs, com frequências entre $5,5 \%{ }^{15}$ e $15,6 \%{ }^{11}$. 0 estudo de Rozelfeld et al..$^{11}$ utilizou-se de critérios de rastreamento semelhantes aos utilizados nessa pesquisa, e a incidência de EAMs também foi próxima. No entanto, no estudo realizado por Mendes et al,,$^{13}$ a incidência de EAM foi menor, fato que pode ser justificado pelo interesse específico dos autores em investigar vários tipos de eventos adversos ocorridos no ambiente hospitalar, não se restringindo aos critérios de rastreamento específicos para os EAMs. A identificação de EAMs requer a utilização de um conjunto de rastreadores específicos relacionados com administração de medicamentos, alterações em exames laboratoriais e mudança no quadro clínico do paciente.

A incidência de eventos adversos é maior em hospitais de ensino quando comparados com os demais. ${ }^{16}$ No entanto, nesse estudo o delineamento não permitiu avaliar associação entre o perfil do hospital e ocorrência de eventos adversos. Cabe destacar que esses hospitais prestam uma assistência de alta complexidade e, geralmente, agregam os pacientes mais graves.

Considerando que se trata de um hospital cardiológico, esperava-se uma alta concentração de pacientes com o diagnóstico de doença isquêmica do coração (DIC). No entanto, o diagnóstico mais frequente de internação hospitalar foi angina, seguida de insuficiência cardíaca, arritmia e, em quarto lugar, o infarto agudo do miocárdio. 0 infarto agudo do miocárdio é o principal componente no grupo de DIC. Trata-se de evento agudo, que sempre requer internação hospitalar e, pela sua magnitude e existência de procedimentos terapêuticos capazes de melhorar o prognóstico do paciente, tem sido apontado como um agravo importante no desenvolvimento de indicadores para 0 monitoramento da qualidade da assistência. ${ }^{14} \mathrm{~A}$ maioria das internações ocorre pelo agravamento de uma doença crônica, como a angina, por exemplo. 0 fato de esse hospital não possuir atendimento de emergência pode explicar em parte o padrão verificado.

A sobrevida livre de EAM foi menor entre as mulheres nos primeiros 30 dias de internação. Sabe-se que mulheres recebem tratamentos menos agressivos do que os homens e apresentam uma probabilidade mais baixa de admissão em unidade coronariana e de serem submetidas ao uso de trombolíticos e angiografia coronária. É possível que mulheres mais jovens com sintomas sugestivos de doenças cardiológicas sejam apreciadas de forma imprecisa pelas equipes de saúde, ou tais pacientes busquem assistência médica tardiamente. ${ }^{17}$

Acredita-se que, no caso específico do hospital estudado, a longa permanência possa ser parcialmente explicada pela necessidade de realização de exames complementares e especializados, ainda durante a internação. Adicionalmente, há situações em que a internação é antecipada ou se prolonga para garantir sua hospitalização. A falta de recursos de custeio e equipamentos quebrados podem ainda gerar aumento da permanência hospitalar. Somando-se aos fatores citados, dificuldades financeiras, familiares e sociais podem contribuir para o adiamento da alta hospitalar.

0 tempo de permanência, comumente utilizado como um indicador de eficiência hospitalar e medida substitutiva de custos, tem sido visto como um indicador relacionado à qualidade do cuidado prestado, embora a natureza desta relação ainda permaneça obscura. ${ }^{18}$ Permanências significativamente mais curtas do que o esperado podem indicar a presença de esforços voltados para a diminuição de custos através da alta prematura dos pacientes, ou seja, baixa qualidade. Por outro lado, tempos de permanência significativamente mais longos do que 0 esperado também podem ser vistos como indicativo de ineficiência administrativa ou baixa qualidade do cuidado prestado, visto que esta permanência pode ser necessária em decorrência de complicações resultantes do cuidado deficiente.

Outro aspecto a ser considerado é o perfil de gravidade dos casos internados, ponto crítico a ser observado na comparação entre hospitais já que, no conjunto, os pacientes podem compor grupos extremamente heterogêneos em relação ao consumo de recursos e riscos clínicos. A gravidade dos casos exerce forte influência sobre o resultado do cuidado prestado e tem sido destacada como um importante fator de confundimento. 0 ajuste de gravidade nesse estudo esteve limitado às variáveis sexo e idade.

A enfermagem atua em todas as etapas do sistema de medicação e é capaz de interceptar uma elevada proporção de erros. 0 processo de prevenção e interceptação de erros previne o agravamento do quadro clínico quando este está diretamente relacionado à qualidade da assistência.

Conhecer a influência do fator tempo na ocorrência de desfechos diversos pode fazer toda diferença. A probabilidade de ocorrência de EAMs, por exemplo, é diretamente proporcional ao tempo de internação. Os enfermeiros devem ser capazes de identificar falhas no sistema de medicação, instituir mudanças nos processos e, sobretudo, propor barreiras de controle e sistemas de vigilância.

\section{CONCLUSÕES}

A alta incidência de EAMs observada sugere que esta seja ainda maior em outros contextos que não partilhem do nível de excelência da instituição pesquisada. A revisão 
retrospectiva de prontuários orientada por critérios explíitos de rastreamento fornece boas indicações da natureza e da incidência dos eventos adversos. No entanto, a disponibilidade de dados confiáveis e a qualidade das informações registradas nos prontuários podem comprometer a detecção do evento adverso.

A realização de estudos que abordem a relação entre o efeito do tempo e a ocorrência de EAM são fundamentais para o monitoramento e a implementação de mecanismos de defesa, barreira e proteção voltadas para a melhoria da segurança do paciente.

\section{REFERÊNCIAS}

1. Cano FG, Rozenfeld S. Adverse drug events in hospitals: a systematic review. Cad Saude Publica. 2009; ( 25 sup 3):S360-S372.

2. Rozenfeld S. Agravos provocados por medicamentos em hospitais do Estado do Rio de Janeiro, Brasil. Rev Saude Publica. 2007 fev; 41(1): 108-15.

3. Tribiño G, Maldonado C, Segura O, Díaz J. Costos directos y aspectos clínicos de las reacciones adversas a medicamentos en pacientes hospitalizados en el servicio de medicina interna de una institución de tercer nivel de Bogotá. Biomédica. 2006 mar; 26(1): 31-41.

4. Aguiar G, Júnior LAS, Ferreira MAM. llegibilidade e ausência de informação nas prescrições médicas: fatores de risco relacionados a erros de medicação. RBPS. 2006; 19 (2): 84-91.

5. Melo ABR, Silva LD. Segurança na terapia medicamentosa: uma revisão bibliográfica. Esc Anna Nery. 2008; 12 (1): 166-72.

6. Zhang M, Holman CDA, Price SD, Sanfilippo FM, Preen DB, Bulsara M. Comorbidity and repeat admission to hospital for adverse drug reactions in older adults: retrospective cohort study. BMJ: Britsh medical journal 2009; 338a: 2752.

7. Gimenes FRE, Teixeira TCA, Silva AEBC, Optiz SP, Mota MLS, Cassiani SHDB. Influência da redação da prescrição médica na administração de medicamentos em horários diferentes do prescrito. Acta Paul Enferm. 2009; 22(4): 380-84.

8. Classen DC, Pestotnik SL, Evans RS, Lloyd JF, Burke JP. Adverse drug events in hospitalized patients. JAMA 1997; 277 (4): 301-306.

9. Newby LK, Hasselblad V, Armstrong PW, Van de Werf F, Marl DB, White HD, Topol EJ, Califf RM. Time-based risk assessment after myocardial infarction. Implications for timming of discharge and applications to medical decision-making. Eur Heart J 2003; 24 (2): 182-89.

10. Rozich JD, Haraden CR, Resar RK. Adverse drug event trigger tool: a pratical methodology for measuring medication related harm. Qual Saf Health Care Med [serial on-line]. 2003 [cited 2007 May 15]. Available from:http://www.ihi.org/lHI/Results/WhitePapers/ IHIGlobalTriggerToolWhitePaper.htm
11. World Health Organization- WHO. Department of Essential Drugs and Medicines. The Uppsala Monitoring Centre. The importance of pharmacovigilance: safety monitoring of medicinal products. Genebra; 2002.

12. Rozenfeld S, Chaves SMC, Reis LGC, Martins M, Travassos C, Mendes W. et al. Efeitos adversos a medicamentos em um hospital público: estudo piloto. Rev Saude Publica. 2008; 43 (5): 887-90.

13. Szklo M, Nieto FJ. Epidemiology: beyond the basics. $2^{\text {nd }}$ ed. Maryland: Aspen Publication; 2006. 495 p.

14. Melo ECP. Infarto agudo do miocárdio no município do Rio de Janeiro: qualidade dos dados, sobrevida e distribuição espacial. [tese]. Rio de Janeiro: Escola Nacional de Saúde Pública, Fundação Oswaldo Cruz; 2004.

15. Mendes W, Martins M, Rozenfeld S, Travassos C. The assessment of adverse events in hospitals in Brazil. Int I Qual Health Care 2009; 21 (4): 279-84.

16. Baker GR, Norton PG, Flintoft V, Blais R, Brown A, Cox J et al. The Canadian Adverse Events Study: the incidence of adverse events among hospital patients in Canada. Can Med Assoc J 2004; 170: 1678-686.

17. Conti RAS, Solimene MC, Luz PL, Benjó AM, Lemos Neto PA, Ramires JAF. Comparison between young males and females with acute myocardial infarction. Arq Bras Cardiol 2002; 79 (5): 518-25.

18. Thomas JW, Guire KE, Horvat GG. Is patient length of stay related to quality of care? Hosp Health Serv Adm. 1997; 42(4): 489-507. 